\title{
Diagnostic Accuracy of Ascitic Fluid Lactoferrin in Diagnosis of Spontaneous Bacterial Peritonitis
}

\author{
Elham.O.Hamd, Ahmed. A.Allam, Abdel-Hady.R. Abdel-Gwad, \\ Esraa.A.mahmoud
}

\begin{abstract}
:
SBP is a frequent and severe complication in patients with cirrhosis and ascites. diagnosticparacentesis is used commonly to investigate the prescence of SBP. The aim of this study was to evaluate Therole of ascitic fluid lactoferrin as reliable biomarker for the presence ofPMNs and detection of SBP in patients with cirrhosis.ascitic fluid lactoferrin level is much higher among patients with SBP compared to those without SBP.The usefulness of ascetic fluid lactoferrin is still uncertain to rule out SBP because the small number of cases.
\end{abstract}

\section{Introduction}

Spontaneous bacterial peritonitis (SBP) is a frequent and severe complication in patients with cirrhosis and ascites . SBP is a bacterial infection that occurs in the absence of an evident intraabdominal and surgically treatable source of infection (Enomotoand Nishiguchi, 2015). Cirrhotic ascites forms as a result of development of portal hypertension, hypoalbuminemia and reduced plasma oncotic pressure which favor the extravasation of fluid from the plasma to the peritoneal fluid (Sheerand Runyon, 2005). Some patients with SBP have symptoms or signs clearly suggestive of peritoneal infection where as others may be completely asymptomatic or with minor symptoms only. Therefore, diagnostic paracentesis is used commonly to investigate the prescence of SBP (Ribeiro et al., 2007). The diagnosis of SPB is based on a polymorphonuclear leukocyte (PMN) count in ascitic fluid of $\geq 250$ cells $/ \mathrm{mm}^{3}$, irrespective of whether the ascitic fluid indicates positive results on bacterial culture (Fernandez and Acevedo, 2015).SPB leads to hospitalization of $10 \%-30 \%$ of cirrhotic patients, and the mortality rate in this group approaches 30\%
(Huang et al., 2015).Lactoferrin is an iron binding protein that is found mainly in external secretions and in PMNs and is released on degranulation (Booneet al., 2013). The measurement of ascitic fluid lactoferrin could provide a reliable biomarker for the presence of PMNs and detection of SBP in patients with cirrhosis as lysis of the PMNs during transport to the laboratory may lead to false negative results (khalifa et al., 2013).

Our study included 6 original articles, 2 responses and editorials to the first published original article, 2 review articles, one conference abstract and one systematic review.

\section{Objectives:}

Collect and analyze multiple research studies to assess the possible role of lactoferrin in diagnosis of SBP.

Strategy and methods of the research:

The strategy of this systematic review was based upon raising some research questions addressing the possible role of lactoferrin in diagnosis of SBP and put a plan to find the best available answers for each. This was done by looking in the literature and critically appraising the available researches in this field. Good quality researches had 
been selected to reach a conclusive answer for each question.

\section{The research questions are:}

1 - Is there an evidence-based value of lactoferrin in diagnosis of SBP?

2- Can cell count using haemocytometer be replaced by lactoferrin in diagnosis of SBP?

3- Is there any evidence about the role of lactoferrin in diagnosis of hepatocellular carcinoma in absence of SBP?

Selection criteria of the available researches:

a) Type of studies:

Randomized clinical trials (RCT) are be the gold standard to obtain the evidence, but such trials are non applicable for our research questions, as there is no intervention done for the cases. So, we searched for comparative and screening researches. Available systematic reviews had been searched for.

b) Time of studies: English language Studies published during last ten years in the following medical databases:

C) Sites visited: Cochrane library, Pubmed, Medline, Sciencedirect, Any other site containing useful information.

d) Keywords used: Lactoferrin, SBP, Liver cirrhosis, Ascites.

e) The topic of interest: Diagnostic accuracy of ascitic fluid lactoferrin in diagnosis of Spontaneous Bacterial Peritonitis.

\section{Results of the study}

In this systematic review, we serached for researches fulfilling our conditions in the internet as mentioned in the "Meterials and methods" section. A total of 23 papers found in the last 10 years. However, we found that; 11 of these papers did not fulfil all of our conditions, either because they used lactoferrin for other purposes than diagnosis of SBP, or they used other methods for dianosis of SBP than ascitic fluid lactoferrin. These 11 papers were then excluded from our study, and only 12 papers included.

zzThe included 12 papers were then analysed and we found that they belong to 6 types of reseaches(Figure 1):

These 6 papers were then thoroughly analysed in our systematic review as follows: 
SOHAG MEDICAL JOURNALDiagnosticAccuracyofAscitic Fluid Lactoferrin in

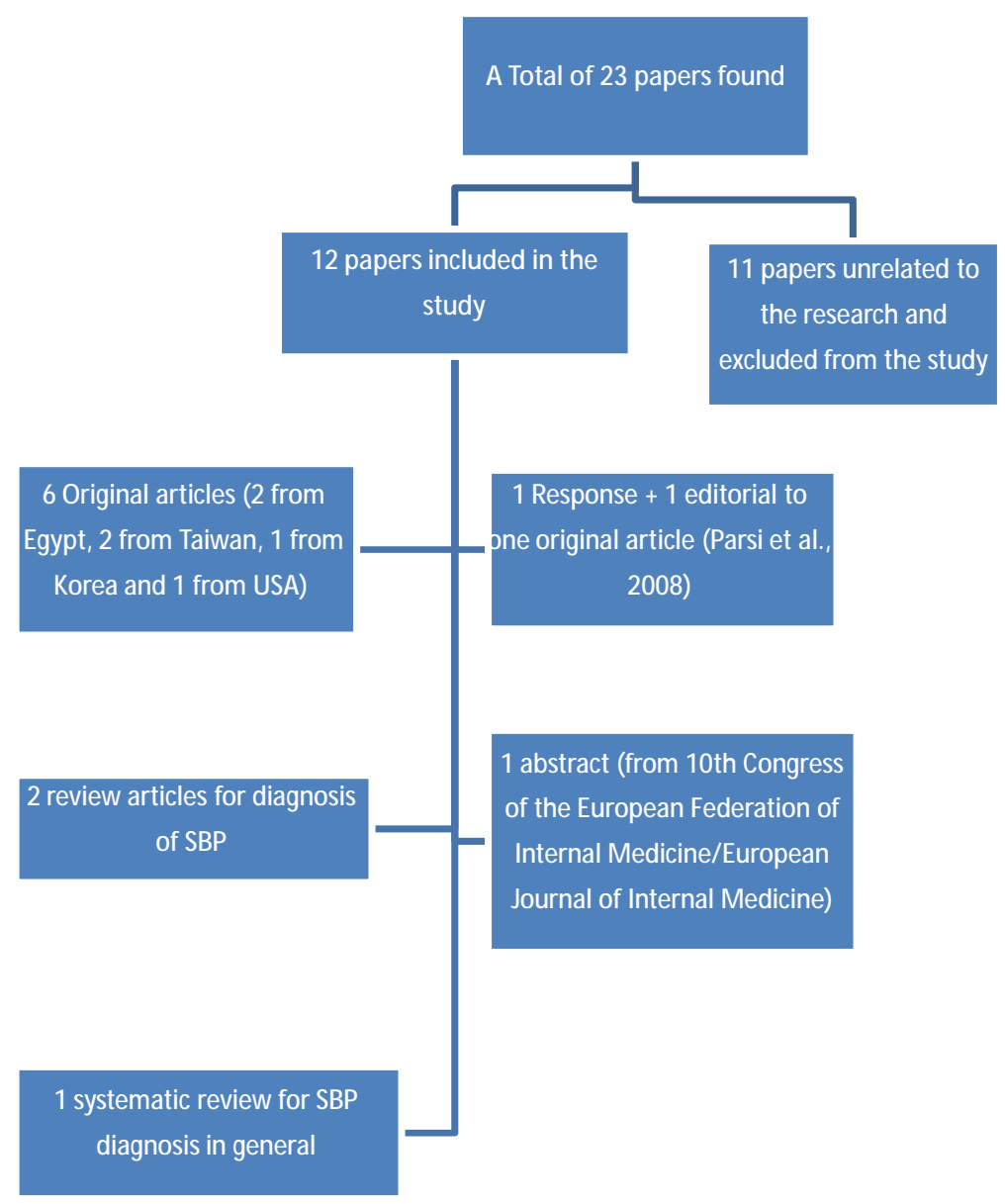

Figure 1. Papers included in our study 
SOHAG MEDICAL JOURNALDiagnosticAccuracyofAscitic Fluid Lactoferrin in

Vol. 23 No.2 July 2018Esraa. A.mahmoud

Table 1. Types and basic data of the included papers in this systematic review

\begin{tabular}{|c|c|c|c|c|}
\hline Serial & Authors & Country & Year & Type of the study \\
\hline $\mathbf{1}$ & Parsi et al & USA & 2008 & Original article \\
\hline $\mathbf{2}$ & Khalifa et al & Egypt & 2013 & Original article \\
\hline $\mathbf{3}$ & Feng et al & Taiwan & 2014 & Original article \\
\hline $\mathbf{4}$ & Wu et al & Taiwan & 2015 & Original article \\
\hline $\mathbf{5}$ & Abulseoudet al & Egypt & 2016 & Original article \\
\hline $\mathbf{6}$ & Lee et al & Korea & 2016 & Original article \\
\hline $\mathbf{7}$ & Thanellas et al & Greece & 2011 & Conference abstract \\
\hline $\mathbf{8}$ & Riggio et al & Italy & 2009 & Response to: \\
\hline $\mathbf{1 1}$ & Riggio et al & Italy & Parsi et al., 2008 \\
\hline $\mathbf{9}$ & Kallwitz & USA & 2009 & Editorial to: \\
\hline & & Parsi et al, 2008 \\
\hline
\end{tabular}

Table 2. Original articles used in this systematic review

\begin{tabular}{|c|c|c|c|c|c|c|c|c|}
\hline Serial & Authors & Country & $\begin{array}{l}\text { No. of } \\
\text { cases }\end{array}$ & $\begin{array}{c}\text { No. of ascetic } \\
\text { samples }\end{array}$ & $\begin{array}{l}\text { Cut off } \\
\text { value }\end{array}$ & 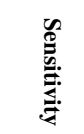 & 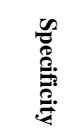 & $\begin{array}{l}\text { Level of } \\
\text { evidence }\end{array}$ \\
\hline 1 & Parsi et al,2008 & USA & 148 & 218 & $242 \mathrm{ng} / \mathrm{ml}$ & $95.5 \%$ & $97 \%$ & III \\
\hline 2 & Khalifa et al,2013 & Egypt & 70 & 70 & $270 \mathrm{ng} / \mathrm{ml}$ & $96 \%$ & $95 \%$ & III \\
\hline 3 & Feng et al,2014 & $\begin{array}{c}\text { Taiwan (and } \\
\text { China) }\end{array}$ & 7 & 8 & - & - & - & IV \\
\hline 4 & Wu et al., 2015 & Taiwan & 22 & 22 & - & - & - & III \\
\hline 5 & Abulseoudet al, 2016 & Egypt & 60 & 60 & $255 \mathrm{ng} / \mathrm{ml}$ & $100 \%$ & $88.9 \%$ & III \\
\hline 6 & Lee et al,2016 & Korea & 102 & 102 & $63 \mathrm{ng} / \mathrm{ml}$ & $91.7 \%$ & $78.2 \%$ & III \\
\hline
\end{tabular}

The 6 original articles were as follows (in chronological order):

1. Parsi et al (2008) from USA

2. Khalifa et al (2013) from Egypt

3. Feng et al (2014) from Taiwan

4. Wu et al (2015) from Taiwan 
5. Abulseoud et al (2015) from Egypt

6. Lee et al (2016) from Kore

Table 3.Meta-analysis of accuracy and cut-off level of AFLAC in the 4 papers with ROC curve analysis (No.1,2,5,6).

\begin{tabular}{|c|c|c|c|c|c|}
\hline Article & Parsi et al & Khalifa et al & Abulseoud et al & Lee et al & Meta-analysis \\
\hline No. of samples & 218 & 70 & 60 & 102 & 450 \\
\hline Cut off value & 242 & 270 & 255 & 63 & 207.52 \\
\hline True positive & 21 & 48 & 30 & 22 & 121 \\
\hline True negative & 187 & 19 & 26.67 & 61 & 293.67 \\
\hline False positive & 6 & 1 & 3.33 & 17 & 27.33 \\
\hline False negative & 1 & 2 & 0 & 2 & 5 \\
\hline $\begin{array}{c}\text { Sensitivity } \\
\text { Specificity }\end{array}$ & $95.45 \%$ & $96.00 \%$ & $100.00 \%$ & $91.67 \%$ & $96.03 \%$ \\
\hline $\begin{array}{c}\text { Positive predictive } \\
\text { value }\end{array}$ & $76.89 \%$ & $95.00 \%$ & $88.90 \%$ & $78.21 \%$ & $91.49 \%$ \\
\hline $\begin{array}{c}\text { Negative predictive } \\
\text { value }\end{array}$ & $99.47 \%$ & $97.96 \%$ & $90.01 \%$ & $56.41 \%$ & $81.57 \%$ \\
\hline $\begin{array}{c}\text { Accuracy } \\
\text { AUC }\end{array}$ & $96.17 \%$ & $90.48 \%$ & $100.00 \%$ & $96.83 \%$ & $98.33 \%$ \\
\hline
\end{tabular}

\section{Discussion}

Ascites is the pathological accumulation of free fluid in the peritoneal cavity. The fluid accumulates because of conditions directly involving the peritoneum (infection, malignancy), or due to other diseases remote from the peritoneum (i.e., liver disease, heart failure, hypoproteinaemia) (Muhammed et al., 2012). Ascites is primarily a transudative fluid with poor opsonic activity which provides a favourable environment for growth of bacteria. SBP rarely occurs without cirrhosis, but cardiac, renal, malignancy, portal vein thrombosis and autoimmune, related infection of ascites has been reported (Scarpellin et al., 2010).SBP is a frequent and severe complication in patients with cirrhosis and ascites. It is a bacterial infection that occurs in the absence of an evident intraabdominal and surgically treatable source of infection (Enomoto and Nishiguchi, 2015). Some patients with SBP have symptoms or signs clearly suggestive of peritoneal infection where as others may be completely asymptomatic or with minor symptoms only. Therefore, diagnostic paracentesis is used commonly to investigate the prescence of SBP (Ribeiro et al., 2007).A variety of factors are associated with the development of SBP including the pathophysiological hallmark: bacterial translocation in an immunocompromised host (Wiest, 2005).Lactoferrin is a red iron-binding whey protein present mainly in external secretions such as breast milk and in polymorphonuclear neutrophils. The presence of lactoferrin in body fluids is proportional to the flux of neutrophils and its assessment can provide a reliable biomarker for inflammation (Sugiyama et al., 2002).In gastrointestinal diseases increased fecal lactoferrin is a sensitive and specific surrogate marker for inflammatory bowel diseases in patients with chronic diarrhea and pain, and ascites lactoferrin can also provide a promising and reliable 
biomarker for bacterial peritonitis (Martins et al., 1995). The measurement of ascitic fluid lactoferrin could provide a reliable biomarker for the presence of PMNs and detection of SBP in patients with cirrhosis as lysis of the PMNs during transport to the laboratory may lead to false negative results (Khalifa et al., 2013).The rationale of our study was to review and assess our knowledge about the value of ascitic fluid lactoferrin level as a diagnostic tool to identify SBP in patients with ascites caused by cirrhosis. Our study included 6 original articles, 2 responses and editorials to the first published original article, 2 review articles, one conference abstract and one systematic review. All of the 6 original articles in cluded in this systematic review showed that ascetic fluid lactoferrin level is much higher among patients with SBP compared to those without SBP (Parsi et al., 2008, Khalifa et al., 2013; Feng et al., 2014; Wu et al., 2015; Abulseoud et al., 2016 and Lee et al., 2016). Four of the 6 original articles used ROC curve analysis to establish a suitable cut-off value for lactoferrin, above which SBP should be suspected. From these 4 articles, 3 gave similar results, with cut off values ranged from 242-270 ng/ml (Parsi et al., 2008, Khalifa et al., 2013; and Abulseoud et al., 2016); while the fourth article showed much lower cut-off value of only $63 \mathrm{ng} / \mathrm{ml}$; with much lower specificity and no better specificity (Lee et al., 2016). The meta-analysis of these 4 articles showed that the cut-off value may be adjusted to around $207 \mathrm{ng} / \mathrm{ml}$, with a sensitivity of over $96 \%$ and a little lower specificity of $91.5 \%$ and an overall accuracy of $93.8 \%$.

\section{Conclusion}

The measurement of ascitic fluid lactoferrin could provide a reliable biomarker for the presence of PMNs and detection of SBP in patient with cirrhosis. ascetic fluid lactoferrin level is much higher among patients with SBP compared to those without SBP.

\section{References}

1. Ashraf $\mathrm{H}$, Beltinger $\mathrm{J}$ and Alam $\mathrm{NH}$ (2007). Evaluation of fecal occult blood test and lactoferrin latex agglutination test in screening hospitalized patients for diagnosis inflammatory and noninflammatory diarrhea in Dhaka, Bangladesh. Digestion 76: 256261, 2007.

2. BooneJ. H., DiPersioJ. R., TanM. J., SalstromS.-J., K. N. Wickham, R. J. Carman, H. R. Totty, R. E. Albert, and D. M. Lyerly (2013)."Elevated lactoferrin is associated with moderate to severe Clostridium difficile disease, stool toxin, and infection. Eur $\mathbf{J}$ ClinMicrobiol Infect Dis. 2013; 32(12): 1517-1523.

3. Dever J.B. and Sheikh M.Y. (2015).spontaneous bacterial peritonitis - bacteriology, diagnosis, treatment, risk factors and prevention. Alimentary pharmacology and therapeutics : Volume 41, Issue 11 June 2015 Pages 1116-1131.

4. Enomoto $H$ and Nishiguchi $S$ (2015). Factors associated with the response to interferon-based antiviral therapies for chronic hepatitis C. World J Hepatol 2015; 7(26):2681-7.

5. Fernandez $J$ and Acevedo $J$ (2015). New antibiotic strategies in patients with cirrhosis and bacterial infection. Expert Rev GastroenterolHepatol 2015; 9(12):1495-500.

6. Huang D., Y. Zhao, Y.Jiang ,Z.Li , w.Yang and G. Chen(2015). "Spontaneous bacterial peritonitis caused by Aeromonascaviae in a patient with cirrhosis." Zhong Nan Da XueXueBao Yi Xue Ban(2015). 40(3): 341-344.

7. Khalifa, N.A., Abdel-Azzez H.A., Hassaneen A. M. and ElShamiE.A(2013)."Ascitic fluid lactoferrin as adiagnostic marker for spontaneous bacterial peritonitis" AfroEgypt J Infect Endem Dis 2013; 3(2): 49-55. 


\section{Min Lee; Kwang-Hyub Han and Sang HoonAhn (2009).}

GastroenterolHepatol. 2009;24(9): 1494-1503.

9. Muhammed H., AslamSaleem S., Alvi A.A. and Hasan S.S (2012). Epidemiology and Symptomatology of Exudative and Transudative type of Ascites in Hospitalized Patients. Pakistan Journal of Pharmacology, 2012; Volume 29, Issue 2, Pages 1-6.

10.Parsi M, Saadeh SN and Zein NN (2008). Ascites fluid lactoferrin for diagnosis of spontaneous bacterial peritonitis. Gastroenterology 2008;135:803-7.

11.Parsi MA, Shen BO and AckkanJP(2004). Fecal lactoferrin for diagnosis of symptomatic patients with ileal pouch-anal anastomosis. Gastroenterology, 2004; 126: 12801286.

12. Ribeiro TC and Kondo $M$ (2007). Evaluation of reagent strips for ascitic fluid leukocyte determination: is it a possible alternative for spontaneous bacterial peritonitis rapid diagnosis? Braz J Infect Dis 2007;11(1): 70-4.

13. Riggio O, Marzano $C$, Angeloni $S$ (2009). Do we really need alternatives to polymorphonuclear cells counting in ascitic fluid? Gastroenterology 2009; 136: 728-9.

14. Runyon B.A. (2013). Introduction to the revised American Association for the Study of Liver Diseases Practice Guideline management of adult patients with ascites due to cirrhosis 2012. Hepatology2013; 57:16511653).

15. Scarpellini E, Valenza $V$ and Gabrielli M.(2010) Intestinal permeability in cirrhotic patients with and without spontaneous bacterial peritonitis: is the ring closed? Am J Gastroenterol 2010; 105: 323-7.

16. Sheer $T A$ and Runyon BA(2005). Spontaneous bacterial peritonitis. Dig Dis 2005; 23(1): 39-46. spontaneous bacterial peritonitis. Gut 2007; 56: 597-9.

17. Sugiyama T, Tsuchida M, Yokota K, Shimodan $M$ and AsakaM(2002). Improvement of long-standing irondeficiency anemia in adults after eradication of Helicobacter pylori infection. Intern Med. 2002; 41: 491494.

18. Wiest R. (2005). Bacterial Translocation ; Department of Internal Medicine I, University Hospital Regensburg, Germany 61 Review Bioscience Microflora, 2005, Vol. 24 (3) 61:90.

19. William W.L. Hsiao, Christine Metz, Davinder P. Singh and Jesse Roth (2008).The Microbes of the Intestine: An Introduction to Their Metabolic and Signaling Capabilities, EndocrinolMetabClin North Am. 2008 Dec; 37(4): 857-871.

20. Wong, F., Blei, A.T., Blendis, L.M. and Thulavath, P.J (2003). A vasopressin receptor antagonist (VPA-985) improves serum sodium concentration in patients with hyponatremia: a multicenter, randomized, placebo controlled trial. Hepatology, 2003; Volume 37, Pages 182-191.

21. Wong CL, Holroyd-Leduc J, Thorpe KE, Straus SE (2008). Does this patient have bacterial peritonitis or portal hypertension? How do I perform a paracentesis and analyze the results?JAMA2008;299:11661178 . 